\title{
The Endoplasmic Reticulum Stress Response Mediates Shikonin-Induced Apoptosis of 5-Fluorouracil-Resistant Colorectal Cancer Cells
}

\author{
Mei Jing Piao ${ }^{1,2, \dagger}$, Xia Han $^{1, \dagger}$, Kyoung Ah Kang ${ }^{1,2}$, Pincha Devage Sameera Madushan Fernando", \\ Herath Mudiyanselage Udari Lakmini Herath ${ }^{1}$ and Jin Won Hyun ${ }^{1,2, *}$ \\ ${ }^{1}$ Department of Biochemistry, Jeju National University College of Medicine, Jeju 63243, \\ ${ }^{2}$ Jeju Research Center for Natural Medicine, Jeju National University, Jeju 63243, Republic of Korea
}

\begin{abstract}
Resistance to chemotherapeutic drugs is a significant problem in the treatment of colorectal cancer, resulting in low response rates and decreased survival. Recent studies have shown that shikonin, a naphthoquinone derivative, promotes apoptosis in coIon cancer cells and cisplatin-resistant ovarian cells, raising the possibility that this compound may be effective in drug-resistant colorectal cancer. The aim of this study was to characterize the molecular mechanisms underpinning shikonin-induced apoptosis, with a focus on endoplasmic reticulum (ER) stress, in a 5-fluorouracil-resistant colorectal cancer cell line, SNU-C5/5-FUR. Our results showed that shikonin significantly increased the proportion of sub- $\mathrm{G}_{1}$ cells and $\mathrm{DNA}$ fragmentation and that shikonin-induced apoptosis is mediated by mitochondrial $\mathrm{Ca}^{2+}$ accumulation. Shikonin treatment also increased the expression of ER-related proteins, such as glucose regulatory protein 78 (GRP78), phospho-protein kinase RNA-like ER kinase (PERK), phospho-eukaryotic initiation factor 2 (elF2 $\alpha$ ), phospho-phosphoinositol-requiring protein-1 (IRE1), spliced X-box-binding protein-1 (XBP-1), cleaved caspase-12, and C/EBP-homologous protein (CHOP). In addition, siRNA-mediated knockdown of CHOP attenuated shikonininduced apoptosis, as did the ER stress inhibitor TUDCA. These data suggest that ER stress is a key factor mediating the cytotoxic effect of shikonin in SNU-C5/5-FUR cells. Our findings provide an evidence for a mechanism in which ER stress leads to apoptosis in shikonin-treated SNU-C5/5-FUR cells. Our study provides evidence to support further investigations on shikonin as a therapeutic option for 5-fluorouracil-resistant colorectal cancer.
\end{abstract}

Key Words: Naphthoquinone, 5-Fluorouracil-resistant colorectal cancer, Apoptosis, Endoplasmic reticulum stress

\section{INTRODUCTION}

Drug resistance is a significant obstacle for successful chemotherapy of colorectal cancer. 5-Fluorouracil (5-FU) is widely used for the treatment of numerous cancers, including colon cancer, despite the low response rate of approximately $20 \%$ in this cancer (Kim et al., 2015). Patients with colorectal cancer also exhibit variable response rates to other anticancer drugs (Mohelnikova-Duchonova et al., 2014).

The endoplasmic reticulum (ER) synthesizes lipids involved in the production of the plasma membrane and provides a non-vascular pathway for lipid transport, as well as enzymes for lipid metabolism reactions (Salvador-Gallego et al., 2017). The functions of the ER also comprise protein folding and as- sembly, vesicle transport, and cellular calcium storage. Therefore, the ER is sensitive to changes in cell homeostasis. Since ER function is essential for cell survival, functional disturbances in this compartment can cause cellular damage and lead to apoptosis (Zhang et al., 2012). For example, disruptions of ER function that alter ER homeostasis lead to the inhibition of protein folding and the accumulation of unfolded proteins. Such disruptions can be caused by modifications in $\mathrm{Ca}^{2+}$ homeostasis or increased demand for protein folding, for example, due to elevated synthesis of proteins in specialized secretory cells or the expression of a misfolded mutant protein (Basseri and Austin, 2012; Dastghaib et al., 2021). Such dysfunction causes various types of proteotoxicities in the ER, collectively referred to as ER stress (Pluquet et al., 2015).

\section{Open Access https://doi.org/10.4062/biomolther.2021.118}

This is an Open Access article distributed under the terms of the Creative Commons Attribution Non-Commercial License (http://creativecommons.org/licenses/by-nc/4.0/) which permits unrestricted non-commercial use, distribution, and reproduction in any medium, provided the original work is properly cited.
Received Jul 14, 2021 Revised Aug 26, 2021 Accepted Sep 1, 2021 Published Online Oct 5, 2021

\section{*Corresponding Author}

E-mail: jinwonh@jejunu.ac.kr

Tel: +82-64-754-3838, Fax: +82-64-702-2687

${ }^{\dagger}$ The first two authors contributed equally to this work. 
To resolve ER stress, a three-pronged signal transduction cascade is activated. The upstream components of the three branches are double-stranded RNA-activated protein kinase (PKR)-like ER kinase (PERK), activating transcription factor 6 (ATF6), and inositol-requiring enzyme 1 (IRE1) (Kim et al., 2016). Once these pathways are activated, phosphorylation of eukaryotic translation initiation factor- $2 \alpha(\mathrm{elF} 2 \alpha)$ results in the downregulation of protein synthesis, while ER functional capacity is improved by upregulating the transcription of genes encoding ER chaperones, protein folding enzymes, and components of the ER-associated degradation system.

Lithospermum erythrorhizon is a medicinal plant that is widely used in traditional oriental medicine (Han et al., 2015; Prasad et al., 2015). The bioactive component in L. erythrorhizon root extract is a naphthoquinone derivative called shikonin, which has been shown to trigger apoptosis in the SNU407 colon cancer cells (Han et al., 2019). Shikonin also has a significant apoptotic effect on cisplatin-resistant human ovarian cells (Shilnikova et al., 2018). While shikonin is known to exert apoptotic effects in cancer cells, including drug-resistant cancers, little is known about the molecular mechanisms underlying these effects. In light of findings, which suggest that ER stress may be involved in drug resistance in colorectal cancer cells, we hypothesized that the functional effects of shikonin in cancer may involve the ER stress response. In this study, we investigated whether shikonin induces apoptosis in colorectal cancer 5-FU-resistant SNU-C5 (SNU-C5/5-FUR) cells via activating the ER stress.

\section{MATERIALS AND METHODS}

\section{Reagents}

Shikonin and antibodies against GRP78, phospho-elF2 $\alpha$, phospho-IRE1, and XBP-1 were purchased from Santa Cruz Biotechnology (Dallas, TX, USA). Thiazolyl blue tetrazolium bromide (MTT), ethylene glycol-bis(2-aminoethylether)$\mathrm{N}, \mathrm{N}, \mathrm{N}$, N'-tetraacetic acid (EGTA), Hoechst 33342, propidium iodide (PI), tauroursodeoxycholic acid (TUDCA), and actin antibodies were purchased from Sigma-Aldrich (St. Louis, MO, USA). Rhod-2 acetoxymethyl ester (Rhod-2, AM) and ER-Tracer Blue-White DPX were purchased from Molecular Probes (Eugene, OR, USA). Antibodies against phosphoPERK, caspase-12, and C/EBP-homologous protein (CHOP) were purchased from Cell Signaling Technology (Beverly, MA, USA). All other chemicals and reagents used were of analytical grade.

\section{Cell culture}

SNU-C5/5-FUR cells were obtained from the Resistant Cell Research Center of Chosun University (Gwangju, Korea). The cells were subcultured twice a week in presence of $140 \mu \mathrm{M}$ 5-FU for over 6 months until they were stably drug resistant (Kang et al., 2014). The cells were cultured at $37^{\circ} \mathrm{C}$ with $5 \%$ $\mathrm{CO}_{2}$, in RPMI-1640 medium (Thermo Fisher Scientific, Grand Island, NY, USA) containing HEPES and supplemented with $10 \%$ heat-inactivated fetal bovine serum (FBS) and antibioticantimycotic solution. The drug resistance of the SNU-C5/5FUR cell line was maintained by treatment with 5-FU once a month.

\section{Cell viability assessment}

The cells were seeded into a 24-well plate at a density of $0.8 \times 10^{5} \mathrm{cell} / \mathrm{s} / \mathrm{mL}$ and incubated for $16 \mathrm{~h}$. They were then treated with shikonin at a concentration of $1,2,3,4,5,6,8,10$, or $15 \mu \mathrm{M}$, or pretreated with an ER stress inhibitor (TUDCA) prior to treatment with shikonin for $30 \mathrm{~min}$. The cells were subsequently incubated at $37^{\circ} \mathrm{C}$ for $48 \mathrm{~h}$, and then $125 \mu \mathrm{L} \mathrm{MTT}$ stock solution $(2 \mathrm{mg} / \mathrm{mL}$ ) was added to each well. After $4 \mathrm{~h}$, the formazan crystals were dissolved in $350 \mu \mathrm{L}$ DMSO, and the absorbance at $540 \mathrm{~nm}$ was measured using a SpectraMax i3X multi-detection microplate reader (Molecular Devices, Sunnyvale, CA, USA) (Piao et al., 2019).

\section{Cell morphology analyses}

Cells were seeded into a $60 \mathrm{~mm}$ culture dish at a density of $0.8 \times 10^{5}$ cells $/ \mathrm{mL}$ and incubated for $16 \mathrm{~h}$. After treatment with $3.3 \mu \mathrm{M}$ shikonin, cells were observed for 1,2 , and 3 days, and changes in cell morphology were documented using a phase contrast inverted microscope (DP71 digital microscope camera, Olympus, Tokyo, Japan). All images were acquired at 20x magnification.

\section{Colony formation assay}

Approximately 100 cells were inoculated into a $35 \mathrm{~mm}$ culture dish and allowed to grow for 2 days. The cells were then treated with $3.3 \mu \mathrm{M}$ shikonin and incubated for 10 days until colonies formed. To aid visualization, the colonies were stained using a Diff-Quik kit (Sysmex, Kobe, Japan) according to the manufacturer's instructions.

\section{Detection of sub- $\mathrm{G}_{1}$ hypodiploid cells}

Flow cytometry analysis was performed after cells were stained with propidium iodide $(\mathrm{PI})$ to assess the proportion of sub- $\mathrm{G}_{1}$ cells with hypodiploid DNA content, which are considered to represent apoptotic cells. Briefly, cells were seeded in 6-well plates in triplicate for each of the control and shikonintreated groups. Twenty hours later, cells were pretreated with $3.3 \mu \mathrm{M}$ shikonin (treated). The cells were harvested after $48 \mathrm{~h}$ and fixed with $70 \%$ ethanol $(1 \mathrm{~mL})$ for $30 \mathrm{~min}$ at $4{ }^{\circ} \mathrm{C}$. Subsequently, cells were washed twice with cold PBS $+2 \mathrm{mM}$ EDTA, to prevent aggregation, and incubated in the dark for $30 \mathrm{~min}$ at $37^{\circ} \mathrm{C}$ in PBS $/ 2 \mathrm{mM}$ EDTA containing PI (final concentration, $100 \mu \mathrm{g} / \mathrm{mL}$ ) and RNase A (final concentration, $100 \mu \mathrm{g} / \mathrm{mL}$ ). The analysis was performed using a FACSCalibur instrument, and the percentage of sub- $\mathrm{G}_{1}$ hypodiploid cells was evaluated using CellQuest and ModFit Software (Becton Dickinson, San Jose, CA, USA).

\section{DNA fragmentation analysis}

Cellular DNA fragmentation was assessed by measuring DNA fragments released into the cell cytoplasm. To facilitate detection, DNA was labeled with the non-radioactive thymidine analog, BrdU. DNA fragments were detected immunologically using an ELISA kit from Roche Diagnostics (Mannheim, Germany), according to the manufacturer's instructions.

\section{Measurement of mitochondrial $\mathrm{Ca}^{2+}$}

Mitochondrial $\mathrm{Ca}^{2+}$ levels were monitored using Rhod-2 AM (Mészáros et al., 2012). Cells were treated with shikonin for 48 $\mathrm{h}$; harvested, washed, resuspended in PBS containing $1 \mu \mathrm{M}$ Rhod-2 AM; and incubated for $15 \mathrm{~min}$ at $37^{\circ} \mathrm{C}$. Subsequently, the cells were washed and suspended in PBS for further anal- 
ysis by flow cytometry. To confirm the flow cytometry results, the cells were seeded in 4-well chambers, and image analysis was conducted by loading cells with Rhod-2 AM for 30 min at $37^{\circ} \mathrm{C}$. After washing, the stained cells were mounted on microscope slides with mounting medium (DAKO, Carpinteria, CA, USA). Images were captured on a confocal microscope using the Laser Scanning Microscope 5 PASCAL software (Carl Zeiss, Jena, Germany).

\section{Nuclear fragmentation analysis by Hoechst 33342 staining}

The cells were seeded at $1.0 \times 10^{5}$ cells $/ \mathrm{mL}$ in medium with or without EGTA. After $16 \mathrm{~h}$ incubation, the cells were treated with shikonin and incubated at $37^{\circ} \mathrm{C}$ for another $48 \mathrm{~h}$. Alternatively, the cells were seeded in a 24 -well plate at $1.0 \times 10^{5} \mathrm{cells} /$ $\mathrm{mL}$. After $16 \mathrm{~h}$ incubation, the cells were treated with TUDCA and incubated for $30 \mathrm{~min}$, followed by treatment with shikonin and incubation at $37^{\circ} \mathrm{C}$ for $48 \mathrm{~h}$. After staining with Hoechst 33342 cell-permeable nuclear counterstain dye for $10 \mathrm{~min}$, nuclear fragmentation (indicating apoptosis) was determined as previously described (Piao et al., 2019).

\section{Western blot analysis}

The harvested cells were washed once with PBS, lysed with RIPA buffer containing protease inhibitors on ice for 20 $\mathrm{min}$, and centrifuged at $14,000 \times \mathrm{g}$ for $10 \mathrm{~min}$. The supernatant was collected, and the Quant-iT ${ }^{\mathrm{TM}}$ protein assay kit was used to determine protein concentrations (Thermo Fisher Scientific). After boiling an aliquot of the lysate $(40 \mu \mathrm{g}$ protein) for $5 \mathrm{~min}$, the proteins were separated by sodium dodecyl sulfate-polyacrylamide gel electrophoresis (SDS-PAGE) based on their molecular weight. The separated proteins were transferred onto nitrocellulose membranes, which were subsequently incubated with primary antibodies against GRP78, phospho-PERK, phospho-elF2 $\alpha$, phospho-IRE1, XBP-1, caspase-12, CHOP, and actin, followed by a horseradish peroxidase-conjugated secondary antibody (Pierce, Rockford, IL, USA). The membranes were incubated with enhanced chemiluminescence detection reagents (Amersham, Little Chalfont, Buckinghamshire, UK) and exposed to X-ray film in the dark to visualize protein bands.

\section{Reverse transcription-polymerase chain reaction (RT-PCR)}

RT-PCR was performed as previously described (Han et al., 2019). The sequences of primers used in this study are as follows: spliced $X B P-1$, forward primer $\left(5^{\prime} \rightarrow 3^{\prime}\right)$ CCTTGTAGTTGAGAACCAGG, reverse primer $\left(5^{\prime} \rightarrow 3^{\prime}\right)$ GGGGCTTGGTATATATGTGG; hGAPDH, forward primer $\left(5^{\prime} \rightarrow 3^{\prime}\right)$ TCAAGTGGGGCGATGCTGGC, reverse primer $\left(5^{\prime} \rightarrow 3^{\prime}\right)$ TGCCAGCCCCAGCGTCAAAG.

\section{Immunocytochemistry}

Cells were seeded on a 4-well chamber glass slide at a density of $1.5 \times 10^{5}$ cells $/ \mathrm{mL}$ and incubated for $16 \mathrm{~h}$. Then, the cells were directly treated with $3.3 \mu \mathrm{M}$ shikonin, or pretreated with $1 \mu \mathrm{M}$ of the ER stress inhibitor TUDCA for $30 \mathrm{~min}$, followed by treatment with $3.3 \mu \mathrm{M}$ shikonin. Protein expression was detected using GRP78 and CHOP antibodies, and ERTracker $^{\mathrm{TM}}$ Blue-White DPX reagent was used for localization. Processing of the slides for immunocytochemistry was performed as previously described (Piao et al., 2015).

\section{Transient transfection of small interfering RNA (siRNA)}

The transfection of SNU-C5/5-FUR cells with control (siControl, Santa Cruz Biotechnology) and CHOP (siCHOP, Bioneer, Seoul, Korea) siRNAs was performed according to a previously described method (Zhang et al., 2013). The cells were subcultured and subjected to siRNA transfection. Knockdown efficiency was assessed by detecting protein expression using Western blotting. After $24 \mathrm{~h}$ transfection, the cells were treated with or without shikonin for $48 \mathrm{~h}$. The role of shikonin in CHOP knockdown cells was examined as follows: PI staining and flow cytometry were used to identify sub- $G_{1}$ hypodiploid cells; DNA fragmentation was determined using a cellular DNA fragmentation ELISA kit (Roche Applied Science, Mannheim, Germany); apoptosis was confirmed using Hoechst 33342 staining reagent; cell viability was detected via MTT analysis.

\section{Statistical analysis}

All experiments were repeated three times, and the values presented are the mean \pm standard error of the mean. Analysis of variance (ANOVA) and Tukey's post-hoc test were performed to analyze the differences between the means. Statistical significance was set at $p<0.05$.

\section{RESULTS}

\section{Shikonin induces apoptosis in 5-FU-resistant colorectal cancer cells}

To determine the effect of shikonin on 5-FU-resistant colorectal cancer cells, we treated SNU-C5/5-FUR cells, into a wide range of shikonin concentrations and determined the effects on cell viability. Our results indicated that shikonin exhibits concentration-dependent cytotoxicity in SNU-C5/5-FUR cells, with significant cytotoxicity evident at concentrations greater than $3 \mu \mathrm{M}$. The calculated $I_{50}$ value was $5.7 \mu \mathrm{M}$ (Fig. $1 \mathrm{~A})$. Based on past experience, it is convenient to observe cell changes when the cell survival rate is approximately $60 \%$ $70 \%$; therefore, a concentration of $3.3 \mu \mathrm{M}$, at which the cell survival rate was $65 \%$, was selected as the optimum concentration for further investigations. After $24 \mathrm{~h}$ treatment with 3.3 $\mu \mathrm{M}$ shikonin, morphological changes were observed in the cells, including some dead cells, although the number of dead cells was not prominent. However, after two days of incubation, a significant number of dead cells was observed, and after three days of incubation, most of the cells appeared to be dead (Fig. 1B). To determine whether shikonin inhibits cell proliferation, a colony formation assay was performed. The results showed that compared with the control group, shikonin significantly inhibited colony formation of SNU-C5/5-FUR cells (CFCs) (Fig. 1C). To confirm that the cytotoxic effect of shikonin was due to its ability to promote apoptosis, we used flow cytometry to measure the proportion of sub- $\mathrm{G}_{1}$ DNA content (considered to represent apoptotic cells) after shikonin treatment. Shikonin significantly increased the rate of apoptosis relative to that in the control group (Fig. 1D). Moreover, shikonin-treated cells had a higher proportion of fragmented DNA content than the control group (Fig. 1E). These results suggested that shikonin effectively inhibits the proliferation of SNU-C5/5-FUR cells by inducing apoptosis. 
A

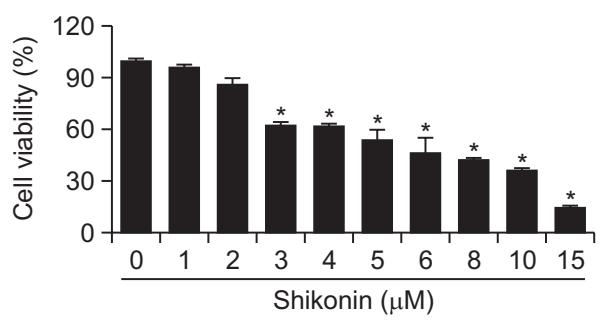

D

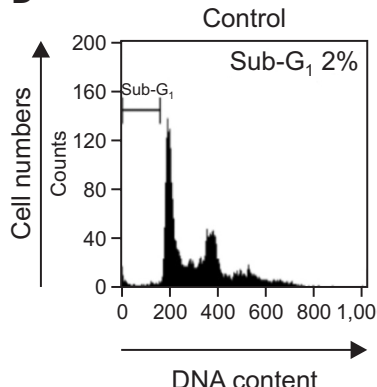

B

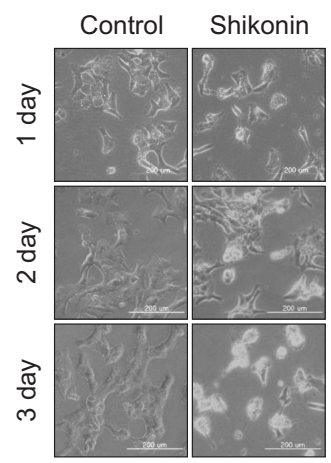

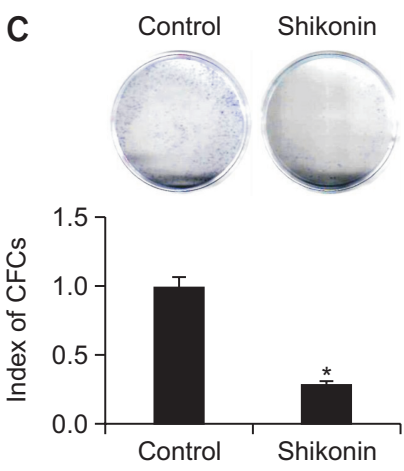

$E$

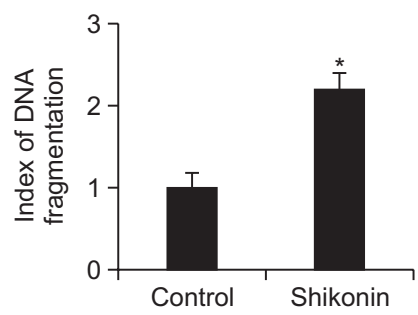

Fig. 1. Shikonin induces apoptotic cell death of SNU-C5/5-FUR cells. (A) Shikonin was added to cells at various concentrations (1, 2, 3, 4, $5,6,8,10$, and $15 \mu \mathrm{M})$. After $48 \mathrm{~h}$, cell viability was determined by MTT assay. *Significantly different from untreated control cells $(p<0.05)$. (B) Changes in cell morphology were detected by phase contrast images (magnification: 20x). (C) Long-term cytotoxic effects of shikonin were investigated using a colony formation assay. The resultant colonies were stained using a Diff-Quik kit. *Significantly different from untreated control cells $(p<0.05)$. (D) Cells treated with or without shikonin were stained with PI and analyzed via flow cytometry. (E) DNA fragmentation was assessed using ELISA. (D, E) *Significantly different from control cells $(p<0.05)$.

\section{Shikonin-induced apoptosis in SNU-C5/5-FUR cells is related to mitochondrial $\mathrm{Ca}^{2+}$ accumulation}

In the mitochondrial matrix, although the accumulation of $\mathrm{Ca}^{2+}$ can stimulate oxidative phosphorylation, high concentrations of $\mathrm{Ca}^{2+}$ can also transmit and amplify apoptotic signals. We observed high levels of fluorescence in the shikonintreated cells, compared to control cells, which was detected using flow cytometry after staining with Rhod-2 AM, a mitochondrial $\mathrm{Ca}^{2+}$-specific dye (Fig. 2A). Images obtained by confocal microscopy also confirmed this result, with shikonintreated cells exhibiting strong red fluorescence, indicating that the accumulation of mitochondrial $\mathrm{Ca}^{2+}$ was higher than that of the control cells (Fig. 2B). To confirm the effect of $\mathrm{Ca}^{2+}$ on shikonin-induced cell apoptosis, we treated SNU-C5/5-FUR cells with shikonin in culture medium with or without EGTA (a $\mathrm{Ca}^{2+}$ chelator) and performed a nuclear fragmentation analysis using Hoechst 33342 staining reagent to detect changes in apoptosis. Compared with the control group, shikonin treatment resulted in a significant increase in apoptosis, but this increase was significantly inhibited in the shikonin+EGTA treatment group (Fig. 2C).

\section{Shikonin increases the levels of ER stress-related proteins and spliced XBP-1 mRNA}

Next, we examined the effect of shikonin on the expression of ER stress-related factors using Western blotting. Compared with the control group, the expression of GRP78, phospho-PERK, phospho-elF2 $\alpha$, phospho-IRE1, spliced XBP-1, cleaved caspase-12, and $\mathrm{CHOP}$ in the shikonin-treated groups increased in a time-dependent manner (Fig. 3A). The activation of phospho-IRE1 leads to unconventional splicing of the mRNA encoding $X B P-1$. The spliced form of $X B P-1$ is translated into a transcription factor that promotes the expression of genes which regulate protein quality in the ER (Xu et al., 2005). Compared with the control cells, the rate of conversion of $X B P-1$ mRNA into its spliced isoform increased in the shikonin-treated cells (Fig. 3B). To confirm this result, we examined the expression of the most representative ER stress marker proteins, GRP78 and CHOP, using immunocytochemistry. Compared with the control group, shikonin significantly upregulated the expression of GRP78 and CHOP in the treated cells (Fig. 3C, 3D).

\section{Downregulation of CHOP attenuates shikonin-induced apoptosis}

It has been reported that overexpression of $\mathrm{CHOP}$ induces apoptosis through the $\mathrm{Bcl}-2$ pathway, and $\mathrm{CHOP}$ has also been shown to regulate apoptosis during ER stress $(\mathrm{Hu}$ et al., 2019). Therefore, we investigated whether $\mathrm{CHOP}$ was required for shikonin-induced apoptosis in SNU-C5/5-FUR cells. We used an siRNA against CHOP (siCHOP) to knock down the expression of CHOP in SNU-C5/5-FUR cells, with a scrambled RNA (siControl) as a negative control, and knockdown was confirmed using Western blot analysis (Fig. 4A). Our flow cytometry analyses showed that shikonin increased the rate of apoptosis in siControl-transfected cells, but this increase was attenuated in cells transfected with siCHOP (Fig. $4 \mathrm{~A})$. This result was confirmed by DNA fragmentation analy- 
A

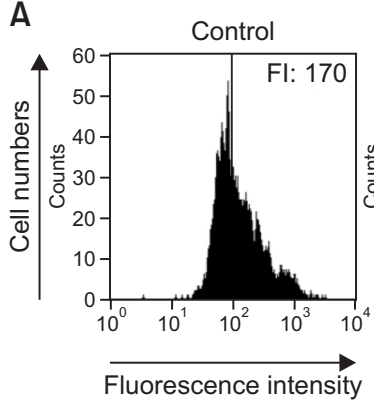

C
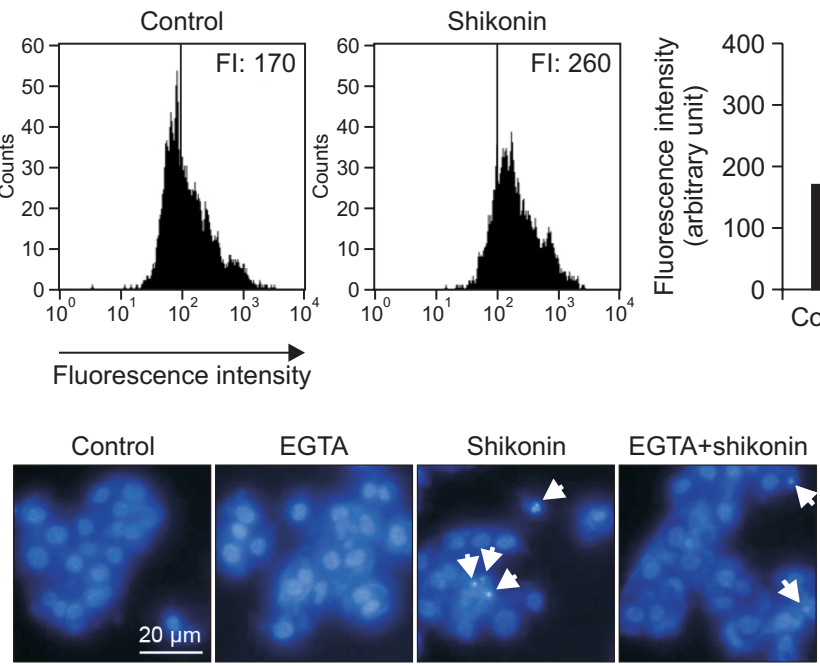

B

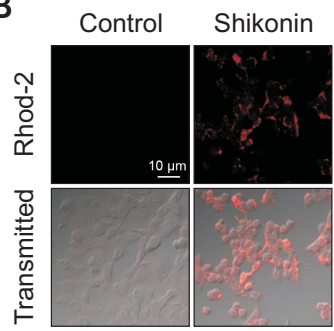

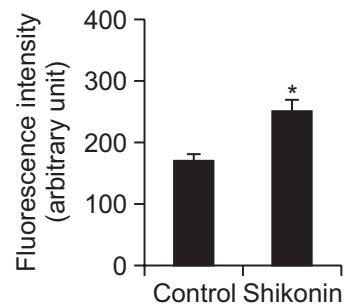

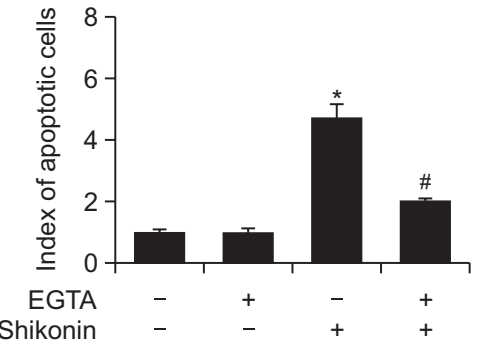

Fig. 2. Shikonin triggers accumulation of mitochondrial $\mathrm{Ca}^{2+}$ in SNU-C5/5-FUR cells. After cells were loaded with the fluorescent probe, Rhod-2 AM, mitochondrial $\mathrm{Ca}^{2+}$ levels were measured via (A) flow cytometry and (B) confocal microscopy. ${ }^{*}$ Significantly different from control cells $(p<0.05)$. (C) Cells were inoculated in culture medium with or without EGTA (500 mM), and after $16 \mathrm{~h}, 3.3 \mu \mathrm{M}$ shikonin was added. After $48 \mathrm{~h}$ incubation, the presence of apoptotic bodies was determined using the Hoechst 33342 nuclear staining method. *'Significantly different from control cells $(p<0.05)$; ${ }^{*}$ significantly different from shikonin-treated cells $(p<0.05)$.

A

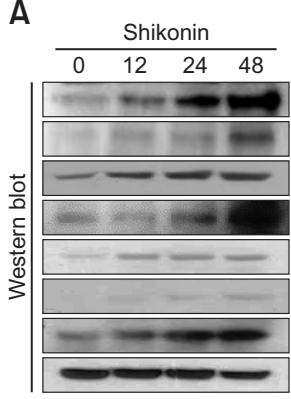

B

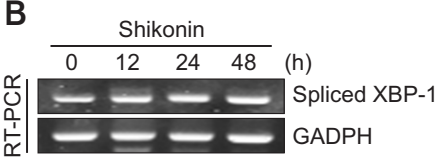

C

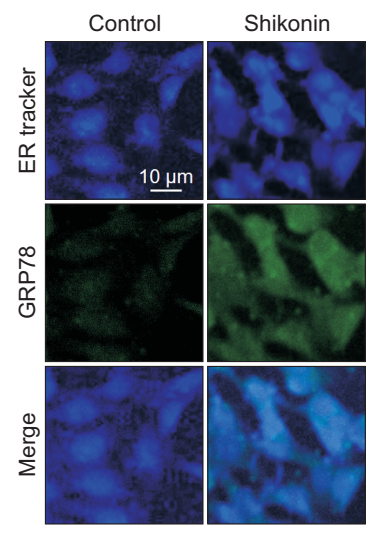

D

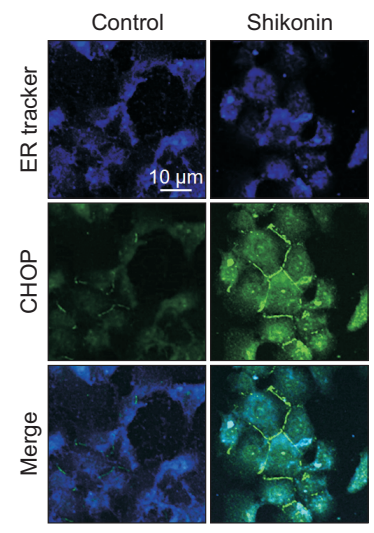

Fig. 3. Shikonin increases the expression of ER stress-related proteins and splicing of XBP-1 mRNA. (A) Cell lysates were subjected to electrophoresis, and GRP78, phospho-PERK, phospho-eIF2 $\alpha$, phospho-IRE1, spliced XBP-1, cleaved caspase-12, and CHOP were detected using Western blotting. Actin was used as a loading control. (B) The level of spliced XBP-1 mRNA was measured using RT-PCR. GAP$\mathrm{DH}$ was used as a loading control. The expression of (C) GRP78 and (D) CHOP proteins were evaluated via immunocytochemistry. The ER-tracker Blue-White DPX probe was used to determine the number and morphology of ER organelles.

sis (Fig. 4B). Moreover, Hoechst 33342 staining for apoptotic bodies also yielded results that were consistent with these findings (Fig. 4C). The results from MTT cell viability assays showed that shikonin inhibited the survival of siControl-transfected cells, but the shikonin-induced decrease in cell numbers was reduced in cells transfected with siCHOP (Fig. 4D). These data demonstrated that CHOP plays an essential role in apoptosis during ER stress induced by shikonin.

\section{Shikonin promotes cell death in SNU-C5/5-FUR cells by activating ER stress}

To further confirm that ER stress is involved in the apoptosis of SNU-C5/5-FUR cells induced by shikonin, we examined the effect of the ER stress inhibitor, TUDCA, in our experiments. As shown in Fig. 5A, TUDCA effectively restored the shikonin-induced decrease in cell viability. Moreover, pretreatment with TUDCA significantly inhibited the emergence of apoptotic bodies (visualized by Hoechst 33342 staining) in the shikonintreated group (Fig. 5B). To confirm this result, we also ana- 
A
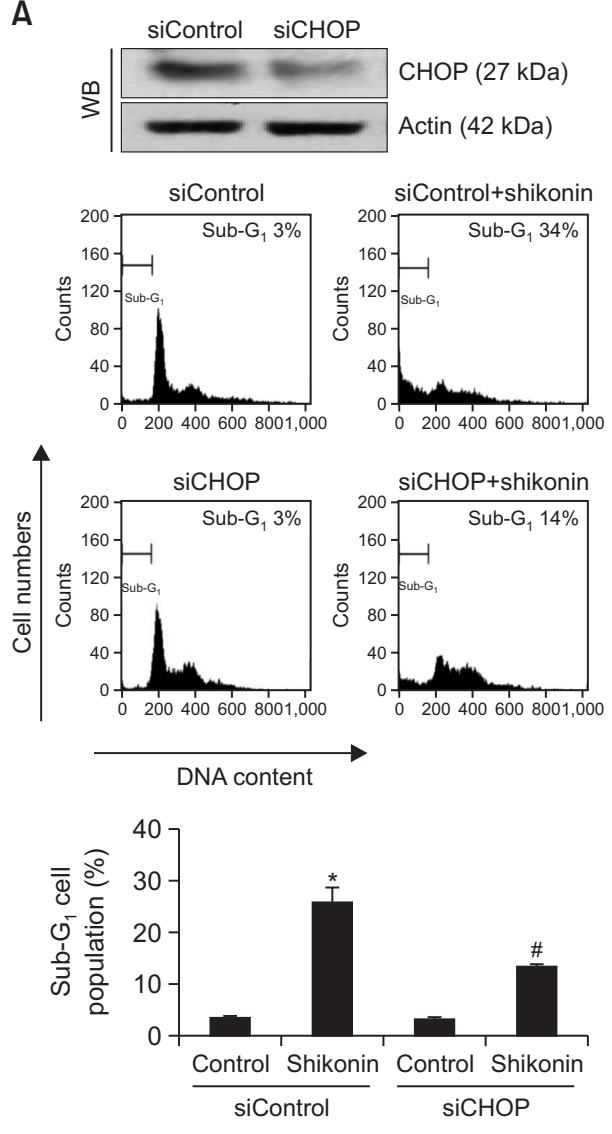

B

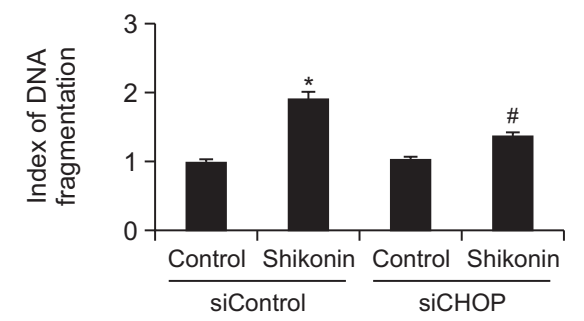

C

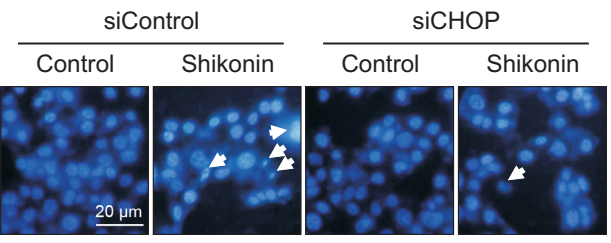

D
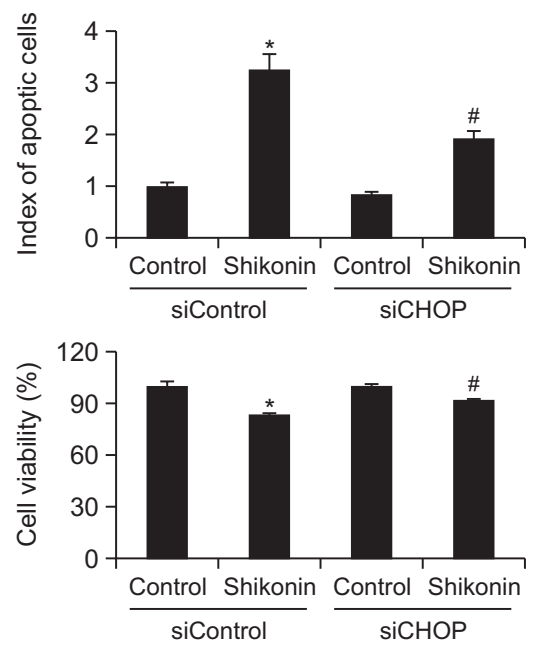

Fig. 4. Knockdown of CHOP attenuates shikonin-induced apoptosis in SNU-C5/5-FUR cells. After confirming the successful transfection of siControl and siCHOP cells via Western blot analysis, the inoculated cells were treated with or without shikonin for $48 \mathrm{~h}$. (A) Cells with apoptotic sub-G $\mathrm{G}_{1}$ DNA content were detected using flow cytometry following PI staining, and (B) DNA fragmentation was quantitated using ELI$\mathrm{SA}$. After $48 \mathrm{~h}$ incubation, (C) the apoptotic bodies were identified via Hoechst 33342 nuclear stain and (D) cell viability was evaluated via MTT analysis. (A-D) *Significantly different from siControl-transfected cells $(p<0.05)$; ${ }^{*}$ significantly different from shikonin-treated, siControltransfected cells $(p<0.05)$.

lyzed the expression of the ER stress marker proteins, GRP78 and $\mathrm{CHOP}$, via immunocytochemistry after TUDCA treatment. TUDCA significantly inhibited the expression of GRP78 and CHOP that were induced by shikonin (Fig. 5C, 5D).

\section{DISCUSSION}

Shikonin has a variety of biological activities, making it an attractive compound. We have previously reported that shikonin induces apoptosis in SNU-407 colon cancer cells by triggering mitochondrial dysfunction and activating the caspase cascade (Han et al., 2019). In that study, we used shikonin at the $\mathrm{IC}_{50}$ concentration of $3.3 \mu \mathrm{M}$ in our experiments. However, in SNU-C5/5-FUR cells, the $\mathrm{IC}_{50}$ value is approximately 5.7 $\mu \mathrm{M}$, which is nearly twice the $\mathrm{IC}_{50}$ value of $3.3 \mu \mathrm{M}$ in the SNU407 cells. Comparing these results, it is apparent that while the sensitivity of shikonin is slightly lower in 5-FU-resistant cells, it still has obvious apoptotic activity in these cells. In this study, we observed that shikonin significantly increased the proportion of SNU-C5/5-FUR cells with apoptotic sub- $\mathrm{G}_{1}$ content and triggered DNA fragmentation.

Generally, the ER stress response is a short-term, homeostasis-linked event that is critical for cell survival, although long-term and severe ER stress can trigger apoptosis through ER stress-specific cell death signals, such as CHOP and caspase-12 (Coker-Gürkan et al., 2015). The ER is the main $\mathrm{Ca}^{2+}$ storage site in cells, and it is worth noting that excessive $\mathrm{Ca}^{2+}$ storage in the ER can result in leaks into the cytoplasm, allowing $\mathrm{Ca}^{2+}$ participation in ER stress-mediated apoptosis (Ryoo, 2015). Here, we used the calcium chelator, EGTA, to verify that treatment with shikonin significantly increased the concentration of $\mathrm{Ca}^{2+}$ in the mitochondrial matrix.

We also studied specific markers of ER stress. Under stressfree conditions, the luminal domain of the ER stress sensor binds to the ER chaperone, binding immunoglobulin protein (BiP), keeping it inactive. When unfolded or misfolded proteins accumulate, BiP preferentially binds to these abnormal proteins and releases the inhibition of PERK, ATF6, and IRE1 (Mei et al., 2013). The cytoplasmic domain of IRE1 possesses 
A
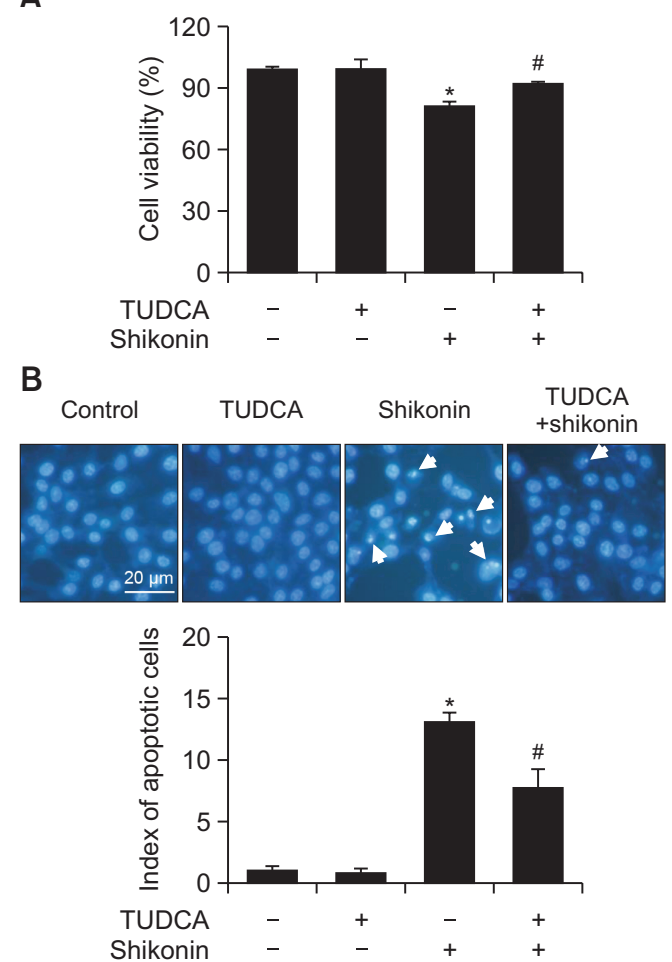

C
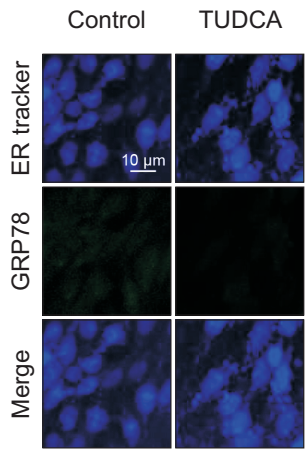

D

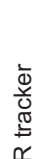

Control

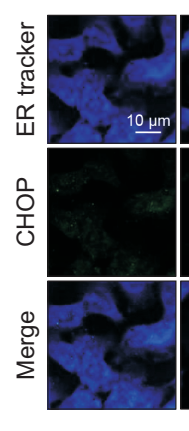

TUDCA

Shikonin

Shikonin $\begin{gathered}\text { TUDCA } \\ \text { +shikonin }\end{gathered}$
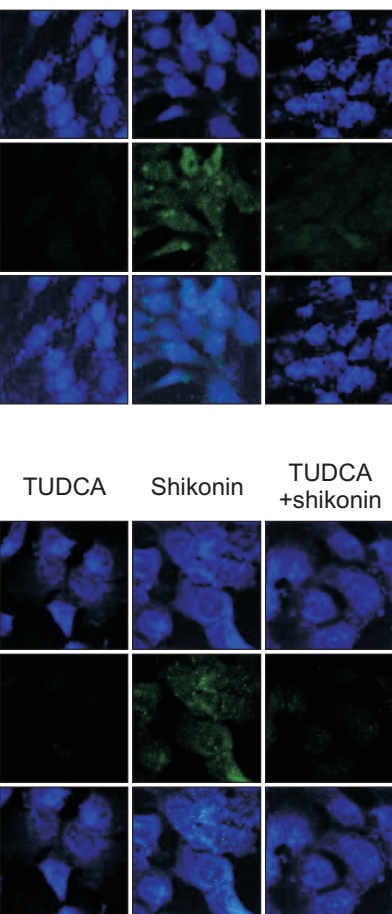

TUDCA
+shikonin
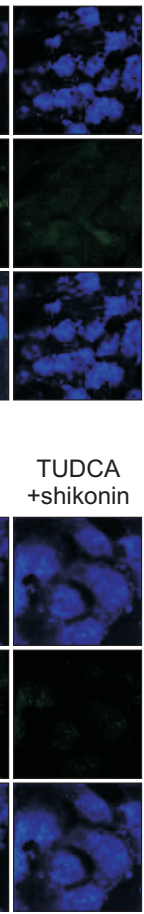

Fig. 5. ER stress inhibitor, TUDCA, attenuates shikonin-induced cell death. Cells were incubated in the presence of $1 \mu \mathrm{M}$ TUDCA for 30 $\mathrm{min}$ and then treated with $3.3 \mu \mathrm{M}$ shikonin. After $48 \mathrm{~h}$ incubation, (A) cell viability was evaluated by MTT analysis, and (B) the presence of apoptotic bodies was determined by Hoechst 33342 nuclear staining. ${ }^{*}$ Significantly different from control cells $(p<0.05)$; ${ }^{*}$ significantly different from shikonin-treated cells $(p<0.05)$. The expression of $(C)$ GRP78 and (D) CHOP proteins was evaluated using immunocytochemistry. The ER-tracker Blue-White DPX probe was used to determine the number and morphology of ER organelles.

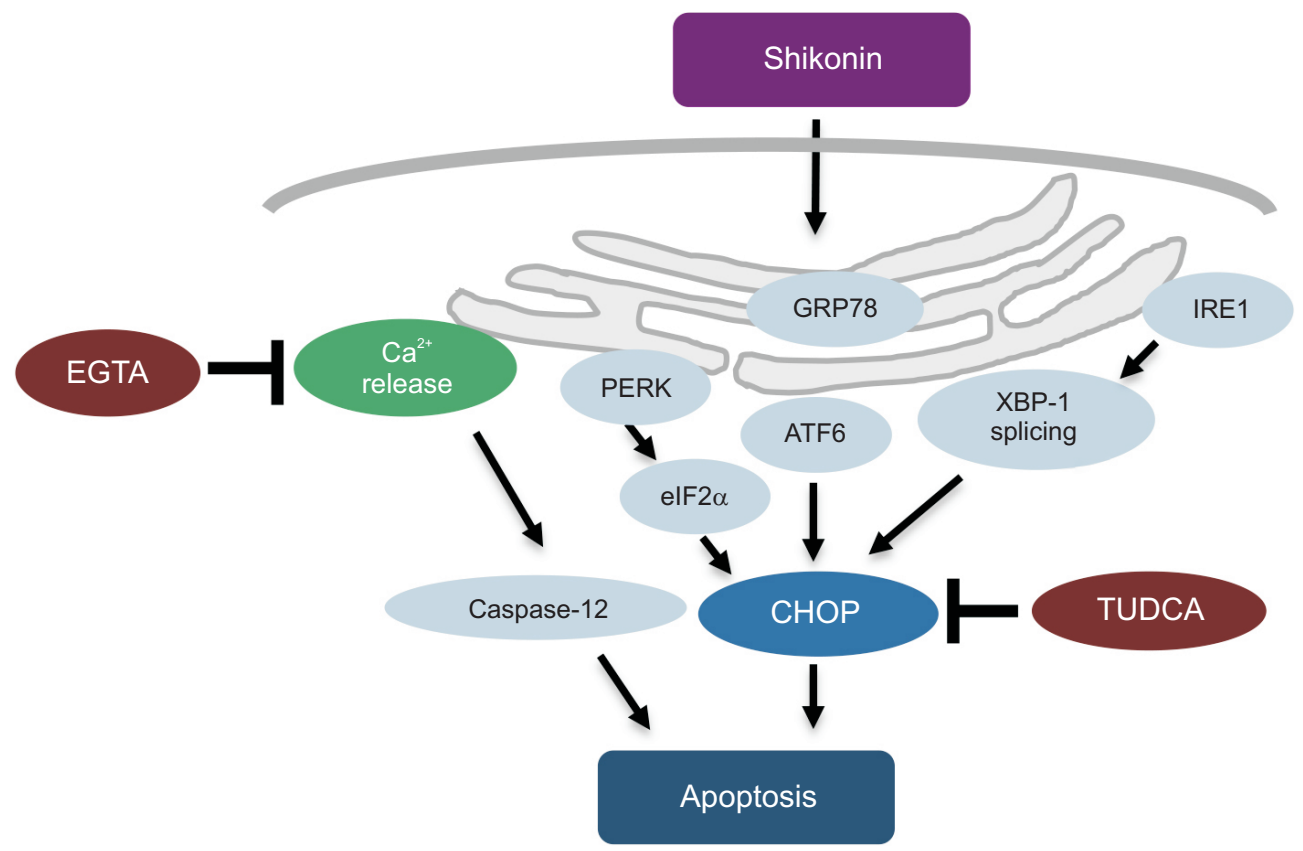

Fig. 6. Diagram of shikonin-induced ER stress signaling resulting in apoptosis. Shikonin induces the expression of ER stress-related proteins, such as GRP78 and CHOP, and releases $\mathrm{Ca}^{2+}$, thereby causing 5-FU-resistant SNU-C5 colorectal cancer cells to undergo apoptosis. The calcium chelator, EGTA, and the ER stress inhibitor, TUDCA, protected cells against ER stressed-apoptotic cell death. 
serine/threonine kinase activity, but the only known substrate of this kinase is IRE1. ER stress-induced homodimerization and trans-autophosphorylation activate IRE1 endoribonuclease activity, allowing it to excise a 26-nucleotide intron from the XBP-1 mRNA to generate the spliced isoform, $X B P-1 \mathrm{~s}(\mathrm{Li}$ et al., 2010). Activated PERK phosphorylates elF2 $\alpha$, thereby reducing protein synthesis and reducing ER protein overload (Yan et al., 2017). Here, we observed that shikonin induced the phosphorylation of PERK, elF2 $\alpha$, and IRE1, as well as XBP-1 splicing, caspase-12 cleavage, and GRP78 and CHOP overexpression, in a time-dependent manner.

Although $\mathrm{CHOP}$ is expressed at very low levels under physiological conditions, it is strongly induced at the transcriptional level under conditions of ER stress (Gotoh et al., 2011). The overexpression of $\mathrm{CHOP}$ promotes growth arrest, and eventually, cell death. Therefore, CHOP plays a very important role in ER stress-induced apoptosis. We observed that siRNAmediated knockdown of CHOP attenuated shikonin-induced apoptosis and cell death. In addition, treatment with the ER stress inhibitor, TUDCA, significantly restored cell viability, and greatly suppressed the emergence of apoptotic bodies due to shikonin exposure. This further supports a role for ER stress in the cytotoxic activity of shikonin in SNU-C5/5-FUR cells (Fig. 6 ). We believe that shikonin is a valuable candidate resource that is worthy of further research as a possible colorectal cancer treatment.

\section{CONFLICT OF INTEREST}

The authors declare that there are no conflicts of interest.

\section{ACKNOWLEDGMENTS}

This research was supported by the 2021 scientific promotion program funded by Jeju National University.

\section{REFERENCES}

Basseri, S. and Austin, R. C. (2012) Endoplasmic reticulum stress and lipid metabolism: mechanisms and therapeutic potential. Biochem. Res. Int. 2012, 841362.

Coker-Gürkan, A., Arisan, E. D., Obakan, P., Akalın, K., Özbey, U. and Palavan-Unsal, N. (2015) Purvalanol induces endoplasmic reticulum stress-mediated apoptosis and autophagy in a time-dependent manner in HCT116 colon cancer cells. Oncol. Rep. 33, 2761-2770.

Dastghaib, S., Kumar, P. S., Aftabi, S., Damera, G., Dalvand, A., Sepanjnia, A., Kiumarsi, M., Aghanoori, M. R., Sohal, S. S., Ande, S. R., Alizadeh, J., Mokarram, P., Ghavami, S., Sharma, P. and Zeki, A. A. (2021) Mechanisms targeting the unfolded protein response in asthma. Am. J. Respir. Cell Mol. Biol. 64, 29-38.

Gotoh, T., Endo, M. and Oike, Y. (2011) Endoplasmic reticulum stressrelated inflammation and cardiovascular diseases. Int. J. Inflam. 2011, 259462.

Han, C. T., Kim, M. J., Moon, S. H., Jeon, Y. R., Hwang, J. S., Nam, C., Park, C. W., Lee, S. H., Na, J. B., Park, C. S., Park, H. W., Lee, J. M., Jang, H. S., Park, S. H., Han, K. G., Choi, Y. W., Lee, H. Y. and Kang, J. K. (2015) Acute and 28-day subacute toxicity studies of hexane extracts of the roots of Lithospermum erythrorhizon in Sprague-Dawley rats. Toxicol. Res. 31, 403-414.

Han, X., Kang, K. A., Piao M. J., Zhen, A. X., Hyun, Y. J., Kim, H. M., Ryu, Y. S. and Hyun, J. W. (2019) Shikonin exerts cytotoxic effects in human colon cancers by inducing apoptotic cell death via the endoplasmic reticulum and mitochondria-mediated pathways. Biomol. Ther. (Seoul) 27, 41-47.

Hu, H., Tian, M., Ding, C. and Yu, S. (2019) The C/EBP homologous protein (CHOP) transcription factor functions in endoplasmic reticulum stress-induced apoptosis and microbial infection. Front. Immunol. 9, 3083.

Kang, K. A., Piao, M. J., Kim, K. C., Kang, H. K., Chang, W. Y., Park, I. C., Keum, Y. S., Surh, Y. J. and Hyun J. W. (2014) Epigenetic modification of Nrf2 in 5-fluorouracil-resistant colon cancer cells: involvement of TET-dependent DNA demethylation. Cell Death Dis. 5, e1183.

Kim, A. Y., Kwak, J. H., Je, N. K., Lee, Y. H. and Jung, Y. S. (2015) Epithelial-mesenchymal transition is associated with acquired resistance to 5 -fluorocuracil in HT-29 colon cancer cells. Toxicol. Res. 31,151-156.

Kim, J. O., Kwon, E. J., Song, D. W., Lee, J. S. and Kim, D. H. (2016) miR-185 inhibits endoplasmic reticulum stress-induced apoptosis by targeting $\mathrm{Na}+/ \mathrm{H}+$ exchanger-1 in the heart. BMB Rep. 49, 208213.

Li, H., Korennykh, A. V., Behrman, S. L. and Walter, P. (2010) Mammalian endoplasmic reticulum stress sensor IRE1 signals by dynamic clustering. Proc. Natl. Acad. Sci. U.S.A. 107, 16113-16118.

Mei, Y., Thompson, M. D., Cohen, R. A. and Tong, X. Y. (2013) Endoplasmic reticulum stress and related pathological processes. J. Pharmacol. Biomed. Anal. 1, 1000107.

Mészáros, G., Szalay, B., Toldi, G., Kaposi, A., Vásárhelyi, B. and Treszl, A. (2012) Kinetic measurements using flow cytometry: new methods for monitoring intracellular processes. Assay Drug Dev. Technol. 10, 97-104.

Mohelnikova-Duchonova, B., Melichar, B. and Soucek, P. (2014) FOLFOX/FOLFIRI pharmacogenetics: the call for a personalized approach in colorectal cancer therapy. World J. Gastroenterol. 20, 10316-10330.

Piao, M. J., Ahn, M. J., Kang, K. A., Kim, K. C., Cha, J. W., Lee, N. H. and Hyun, J. W. (2015) Phloroglucinol enhances the repair of UVB radiation-induced DNA damage via promotion of the nucleotide excision repair system in vitro and in vivo. DNA Repair 28, 131-138.

Piao, M. J., Kang, K. A., Zhen, A. X., Fernando, P. D. S. M., Ahn, M. J., Koh, Y. S., Kang, H. K., Yi, J. M., Choi, Y. H. and Hyun, J. W. (2019) Particulate matter 2.5 mediates cutaneous cellular injury by inducing mitochondria-associated endoplasmic reticulum stress: protective effects of ginsenoside Rb1. Antioxidants 8, 383 .

Pluquet, O., Pourtier, A. and Abbadie, C. (2015) The unfolded protein response and cellular senescence. A review in the theme: cellular mechanisms of endoplasmic reticulum stress signaling in health and disease. Am. J. Physiol. Cell Physiol. 308, 415-425.

Prasad, R. G., Choi, Y. H. and Kim, G. Y. (2015) Shikonin isolated from Lithospermum erythrorhizon downregulates proinflammatory mediators in lipopolysaccharide-stimulated BV2 microglial cells by suppressing crosstalk between reactive oxygen species and NF$\kappa B$. Biomol. Ther. (Seoul) 23, 110-118.

Ryoo, H. D. (2015) Drosophila as a model for unfolded protein response research. BMB Rep. 48, 445-453.

Salvador-Gallego, R., Hoyer, M. J. and Voeltz, G. K. (2017) Snapshot: functions of endoplasmic reticulum membrane contact sites. Cell 171, 1224-1224.e1.

Shilnikova, K., Piao, M. J., Kang, K. A., Ryu, Y. S., Park, J. E., Hyun, Y. J., Zhen, A. X., Jeong, Y. J., Jung, U., Kim, I. G. and Hyun, J. W. (2018) Shikonin induces mitochondria-mediated apoptosis and attenuates epithelial-mesenchymal transition in cisplatin-resistant human ovarian cancer cells. Oncol. Lett. 15, 5417-5424.

Xu, C., Bailly-Maitre, B. and Reed, J. C. (2005) Endoplasmic reticulum stress: cell life and death decisions. J. Clin. Invest. 115, 2656-2664.

Yan, F., Cao, S., Li, J., Dixon, B., Yu, X., Chen, J., Gu, C., Lin, W. and Chen, G. (2017) Pharmacological inhibition of PERK attenuates early brain injury after subarachnoid hemorrhage in rats through the activation of Akt. Mol. Neurobiol. 54, 1808-1817.

Zhang, R., Chung, Y., Kim, H. S., Kim, D. H., Kim, H. S., Chang, W. Y. and Hyun, J. W. (2013) 20-O-( $\beta$-D-glucopyranosyl)-20(S)protopanaxadiol induces apoptosis via induction of endoplasmic 
Piao et al. The Involvement of ER Stress in Shikonin-Induced Apoptosis

reticulum stress in human colon cancer cells. Oncol. Rep. 29, 1365-1370.

Zhang, R., Piao, M. J., Kim, K. C., Kim, A. D., Choi, J. Y., Choi, J. and Hyun, J. W. (2012) Endoplasmic reticulum stress signaling is involved in silver nanoparticles-induced apoptosis. Int. J. Biochem. Cell Biol. 44, 224-232. 